\title{
— "Radial" approach to high risk pulmonary embolism - transcubital catheter directed intervention as a first-line therapy in patients with clinically massive and submassive pulmonary embolism
}

\begin{abstract}
Lovel Giunio*,
Jakša Zanchi,

Anteo Bradarić,

Mislav Lozo
\end{abstract}

University of Split School of Medicine, University Hospital Centre Split, Split, Croatia
KEYWORDS: catheter-based therapies, cubital approach, pulmonary embolism, thrombus fragmentation, thrombolysis.

CITATION: Cardiol Croat. 2016;11(3-4):133. | DOI: http://dx.doi.org/10.15836/ccar2016.133

*ADDRESS FOR CORRESPONDENCE: Lovel Giunio, Klinički bolnički centar Split, Šoltanska 1, HR-21000 Split, Croatia. / Phone: +385-99-203-9999 / E-mail: lovelgiunio@hotmail.com

ORCID: Lovel Giunio, http://orcid.org/0000-0002-1268-5698 • Anteo Bradarić, http://orcid.org/0000-0002-9843-6309 Jakša Zanchi, http://orcid.org/0000-0003-2700-2121 • Mislav Lozo, http://orcid.org/0000-0001-7530-4760

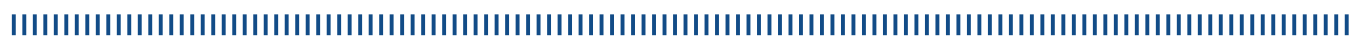

Goal: Despite the guidelines, more than two-thirds of patients with massive/high risk pulmonary embolism (PE) do not receive thrombolytic therapy ${ }^{1,2}$. Submassive/intermediate risk PE, as defined by right ventricular failure and/or NT-proBNP/troponin elevation, which can result in life-threatening deterioration/sequel is treated with thrombolytic therapy even less frequently. The main reason, that thrombolytics are used infrequently despite the potential clinical benefit of rapid clot lysis, is respect for significant bleeding complications, since nonpathologic thrombi are also lysed. The reluctance of majority of treating physicians to actually use thrombolytic therapy in everyday praxis persist and is matched only by zeal to publish successfully treated cases by minority of interventional colleagues. "Radial" - transcubital approach to high risk pulmonary embolism and subsequent catheter directed intervention (CDI) is proposed as a realistic alternative to both routinely used heparin therapy and surgical embolectomy, that can with modifications even be used in the case of contraindications for thrombolytic therapy. The goal of this presentation is to present a real-life center experience of the feasibility and safety of CDI including protocol for transcubital CDI as a first-line therapy in patients with clinically massive/high risk and submassive/intermediate risk PE.

Patients and Methods: From March 2011 throughout December 2015, 29 patients with massive or submassive PE underwent a CDI at the University Hospital Centre Split, Croatia. CDI involved mechanical catheter fragmentation and the application of adjuvant thrombolytic therapy through a pigtail catheter positioned in the pulmonary artery (PA) or rheolytic thrombectomy (RT).

Results: A total of 29 patients were enrolled in the study (15 men, 51.7\%; 14 women, 48.3\%). Clinical success was achieved in 26 of 29 cases (89.6\%). There were no major procedural complications (0\%). The 90-day survival rate was $89.6 \%$.

Conclusion: "Radial" - transcubital CDI in patients with clinically massive or submassive PE is feasible and safe. In experienced centers it should be considered as a first line treatment.

RECEIVED:

February 10, 2016

ACCEPTED:

February 20, 2016

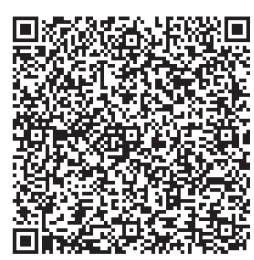

1. Kucher N, Rossi E, De Rosa M, Goldhaber SZ. Prognostic role of echocardiography among patients with acute pulmonary embolism and a systolic arterial pressure of 90 mm Hg or higher. Arch Intern Med. 2005;165(15):1777-81. DOI: http://dx.doi.org/10.1001/archinte.165.15.1777

2. Lin BW, Schreiber DH, Liu G, Briese B, Hiestand B, Slattery D, et al. Therapy and outcomes in massive pulmonary embolism from the Emergency Medicine Pulmonary Embolism in the Real World Registry. Am J Emerg Med. 2012;30(9):1774-81. Dol: http://dx.doi.org/10.1016/j.ajem.2012.02.012 